\title{
Lebenswege jüdischer und aus dem Judentum stam- mender Urologen während des Nationalsozialismus
}

\author{
The Biographies of Jewish Urologists during National Socialism in Germany
}

\section{Bibliografie}

DOI http://dx.doi.org/

10.1055/s-0030-1256217

Akt Dermatol 2011; 37:

130-132 @ Georg Thieme

Verlag KG Stuttgart · New York

ISSN 0340-2541

Korrespondenzadresse

Julia Bellmann

Glasewaldtstraße 20

01277 Dresden

julia_bellmann@gmx.de

\section{Zusammenfassung \\ $\nabla$}

Während der Zeit des Nationalsozialismus 1933 1945 kam es, wie auch in den anderen Fachgesellschaften, in der Urologie zu einer schnellen Anpassung an die herrschenden politischen Verhältnisse. Trotz eines sehr hohen Anteils an jüdischen und jüdisch-stämmigen Urologen ging diese Anpassung nahezu widerstandlos vonstatten. Bereits 1933 erfolgte mit der Gründung der Gesell-

\section{"Er ging nicht freiwillig“ \\ $\nabla$}

Die Deutsche Gesellschaft für Urologie (DGU) hat 2009 zusammen mit Medizinhistorikern aus Ulm (H. Fangerau, M. Krischel), Dresden (A. Scholz, J. Bellmann) und Wien (M. Skopec, F. Butta-Bieck) ein Projekt zur Erforschung der Geschichte der Urologie während des Nationalsozialismus in Deutschland begonnen. Unter der Leitung des Archivars der DGU, D. Schultheiss, und des Vorsitzenden des Arbeitskreises für Geschichte der Urologie, F. Moll, soll dabei sowohl die Täterseite als auch die Opferseite untersucht werden.

Im Rahmen einer Arbeitstagung der Arbeitsgemeinschaft Dermatologie und Venerologie der DDG im Zusammenhang mit der Fortbildungswoche in München wurde im Juli 2010 eines der beiden Teilprojekte vorgestellt: „Lebenswege jüdischer und aus dem Judentum stammender Urologen während des Nationalsozialismus“. Dieses Teilprojekt verfolgt das Ziel, die Schicksale der urologisch tätigen Ärzte aufzuklären, die nach der Machtergreifung der NSDAP im Januar 1933 aufgrund ihrer nichtarischen Herkunft an der Ausübung ihres Berufes gehindert oder stark eingeschränkt waren. Der Verlust der wirtschaftlichen Grundlage, die Verdrängung aus Forschung und Lehre sowie die gesellschaftliche Ausgrenzung der jüdischen bzw. aus dem Judentum stammen- schaft Reichsdeutscher Urologen (GRU) die Gleichschaltung, die Konsequenz war die Verdrängung von jüdischen Kollegen in Praxis, Forschung und Lehre. Die Biografien der davon betroffenen Urologen sind bisher oft nur unzureichend bekannt. Die Klärung der einzelnen Schicksale der nicht arischen Mediziner ist ein Teilaspekt zur Erforschung der Urologie während des Nationalsozialismus.

den Urologen führten zur weitreichenden Veränderung im Fach [1].

Am Anfang des Projektes stand die Frage nach dem zahlenmäßigen Verhältnis von arischen Urologen zu ihren nichtarischen Kollegen. Durch die Auswertung der Reichsmedizinalkalender der Jahre 1933, 1935 und 1937 konnte eine Gesamtzahl von 866 Ärzten für diese 5 Jahre erfasst werden, die sich in der Selbstauskunft zur Erhebung des Kalenders als Urologe bzw. urologisch tätig bezeichneten. Bisher konnte von dieser Gesamtzahl von 866 eine Gruppe von 240 Medizinern ermittelt werden, die sich zum Judentum bekannten bzw. aus dem Judentum stammten ( $\bullet$ Abb. 1).

Das macht einen Prozentsatz von 28\% aus. Der Vergleich zu anderen Fachdisziplinen belegt den unterschiedlich hohen Anteil jüdischer Mediziner in den verschiedenen Fachgebieten. Bei den Pädiatern liegt der Anteil bei 50\% und ist damit der höchste Anteil von jüdischen Ärzten innerhalb einer Fachrichtung [2]. Bei den Dermatologen beträgt der Anteil jüdischer und aus dem Judentum stammender Kollegen 25\% [3,4]. Damit stellen die Urologen die zweithäufigste Gruppe jüdischer Mediziner in den Spezialfächern dar. Die Gruppe von 240 jüdischen und jüdischstämmigen Urologen war der Ausgangspunkt für die genauere Untersuchung des beruflichen Werdeganges und ihres Schicksales während des Nationalsozialismus. 


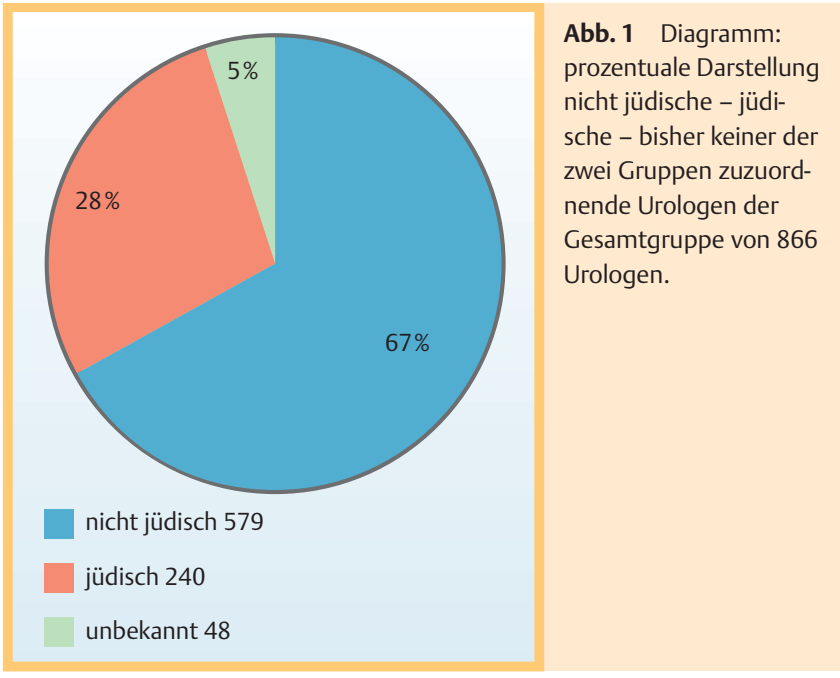

Bedingt durch die junge Fachgeschichte der Urologie, erst seit 1924 konnte man den Facharzt für Urologie erlangen, kam es oftmals zu Überschneidungen mit den vier Quellgebieten der Urologie: der Dermato-Venerologie, der Chirurgie, der Inneren Medizin und der Gynäkologie. Mediziner in den genannten Fachgebieten waren neben ihrer primären fachlichen Orientierung aus der Diagnostik und Mitbehandlung von Erkrankungen aus dem Bereich der Urologie mit diesen speziellen Krankheitsbildern konfrontiert. Innerhalb der zu untersuchenden Personengruppe von 240 jüdischen Urologen stellt die Kombination mit der DermatoVenerologie zahlenmäßig mit 183 (77\%) die stärkste Fraktion ( Abb. 2). Die Häufigkeit der Geschlechtskrankheiten und die Behandlung von Folgezuständen dieser Erkrankungen bedingten die Vielzahl der Betreuung dieser Krankheiten durch Hautärzte. Bereits kurz nach der Machtergreifung 1933 wurde die berufliche Situation für die jüdischen Ärzte durch die ersten Gesetze schwierig. Durch die Gleichschaltung der Ärzteverbände im März 1933 und den beginnenden Boykott von jüdischen Ärzten im darauffolgenden Monat und die ersten Entzüge von Kassenzulassungen wurden sie in der Ausübung ihrer medizinischen Praxis deutlich oder sogar ganz behindert. Hinzu kam das Gesetz zur Wiederherstellung des Berufsbeamtentums, das für viele jüdische Urologen den Verlust ihrer Stellungen in der Lehre oder im Bereich beamteter Positionen im Gesundheitswesen bedeutete. Bereits im ersten Jahr kam es damit zu starken Einschnitten und Umwälzungen in der medizinischen Praxis, Lehre und Forschung. Durch den hohen Prozentsatz an jüdischen Urologen hatte dies weitreichende Veränderungen in dem jungen Fachgebiet der Urologie zur Folge. Jüdische Professoren verloren ihre Lehrberechtigung, so z.B. der Berliner Urologe Eugen Joseph (18791933). Medizinische Gremien und Vorstände wurden ausgewechselt, Praxen mussten geschlossen werden, Kassenzulassungen wurden entzogen, Promotionen und Habilitationen wurden verweigert. Außerdem durften keine Approbationen für jüdische Ärzte mehr erteilt werden.

Trotz dieser Beschränkungen durften die nicht arischen Ärzte noch weiter behandeln. Nach dem Erlass der 4. Verordnung des Reichsbürgergesetzes im Juli 1938 wurde den jüdischen Medizinern die Approbation entzogen und nur noch einige wenige durften unter der Bezeichnung Krankenbehandler ausschließlich jüdische Patienten betreuen. Bei den Urologen sind zurzeit 25 Personen bekannt, die diesen Status erhielten.

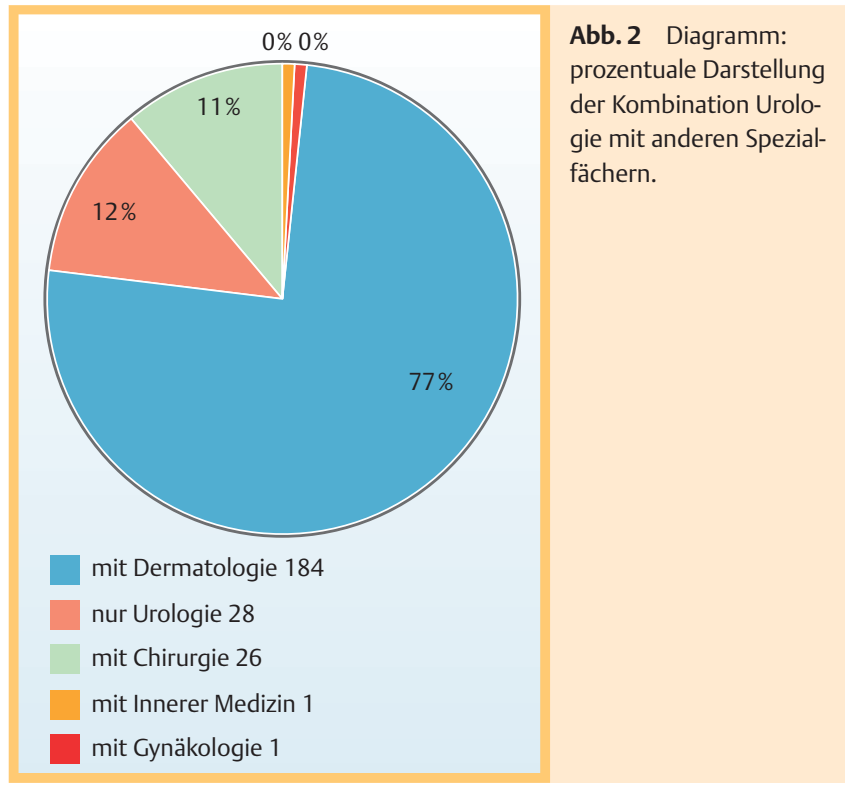

Tab. 1 Emigrationsländer mit der Anzahl der Emigranten.

\begin{tabular}{lc|}
\hline USA & $\mathbf{5 6}$ \\
\hline Palästina & 24 \\
\hline Großbritannien & 12 \\
\hline Unbekanntes Ziel & 6 \\
\hline Frankreich & 5 \\
\hline Holland & 4 \\
Ägypten & 3 \\
\hline Südafrika, Schweden, China & Je 2 \\
Belgien, Siam, Chile, Mandschuko, Kanada, Bolivien, & Je 1 \\
Brasilien, Uruguay, Mexiko, Ecuador &
\end{tabular}

Die Entwicklungen seit 1933 führten zu den unterschiedlichsten Reaktionen der jüdischen Ärzte. Für viele stellte sich die Frage: Emigration oder Bleiben? 127 nicht arische Urologen entschieden sich für die Emigration. Die Hauptemigrationsländer waren die USA, Großbritannien und Palästina ( Tab. 1).

Zwei Beispiele für Emigranten sind Ernst Sklarz und Otto Schlein. Der in Dermato-Venerologie, Urologie und Gynäkologie ausgebildete Ernst Sklarz (1894-1975) hatte von 1930-1936 als Direktor der Dermatologischen Abteilung der Poliklinik am Alexanderplatz in Berlin gearbeitet ( $\bullet$ Abb. 3). Außerdem war er Oberarzt am Krankenhaus der jüdischen Gemeinde in Berlin, bis er im September 1936 nach England emigrierte. Im darauffolgenden Jahr legte er an der Medizinischen Fakultät von Wales das britische Examen ab. Später leitete er seit 1953 die Dermatologische Abteilung des New Cross General Hospital in London. Das Emigrationsland Holland führte den Magdeburger Dermato-Urologen Otto Schlein (1895-1944) in die endgültige Katastrophe. Er emigrierte 1936 mit seiner Familie in die Niederlande. Dort approbierte er 1938 erneut und promovierte nochmals. Doch 1942 wurde er in Amsterdam von der Gestapo verhaftet und kam über mehrere Zwischenstationen im September 1944 mit Frau und Tochter nach Auschwitz, wo er im Oktober des Jahres ermordet wurde [5].

Die Emigration war immer ein Wagnis, bedeutete sie doch einen absoluten Neubeginn unter oftmals stark erschwerten Bedingungen. Die wirtschaftliche Ungewissheit, Sprachbarrieren und weitere Hindernisse beim Neuanfang ließen besonders die älteren Ärzte von einer Auswanderung Abstand nehmen. 


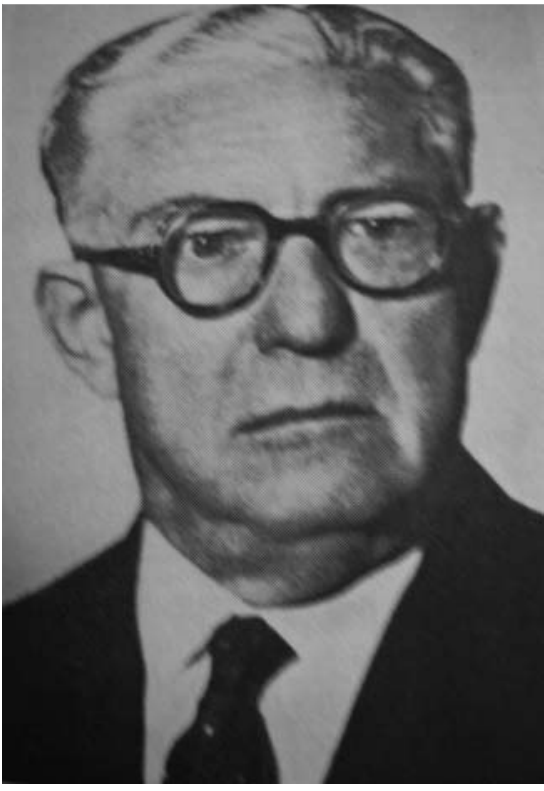

Abb. 3 Ernst Sklarz (1894-1975).

Tab. 2 Bisher geklärte Schicksalswege.

\begin{tabular}{lr} 
Emigration & $\mathbf{1 2 7}$ \\
\hline Deportation & 37 \\
\hline Bekannte Suizide & 10 \\
\hline Verstorben & 29 \\
\hline In Deutschland überlebt & 5
\end{tabular}

Die Urologen, die in Deutschland blieben, hatten unterschiedliche Schicksalswege ( $\bullet$ Tab. 2).

Einige waren vor den Deportationen durch ihren Status als Mischling oder durch einen arischen Ehepartner geschützt. 39 Personen verstarben, wobei bei 10 ein Suizid als gesichert gilt. 37 Personen wurden deportiert, wovon nur 1 überlebt hat. In Deutschland überlebten 5 Urologen. 4 von ihnen blieben in Deutschland, 1 wechselte später nach Großbritannien.

Eine Ärztin, die den Selbstmord wählte, war Dora Gerson (1884 1941). Sie hatte während des 1 . Weltkrieges angefangen im Krankenhaus Friedrichstadt/Dresden zu arbeiten und ließ sich 1926 als Fachärztin für Dermatologie und Urologie in Dresden nieder. Nach dem Entzug ihrer Kassenzulassung 1934 behandelte sie noch knapp ein Jahr Patienten auf privater Basis. 1937 ging sie als Hauswirtschaftslehrerin an die jüdische Gartenbauschule nach Hannover-Glinden. 1940 erhielt sie ihre Zulassung als Krankenbehandlerin. An ihrem 57. Geburtstag 1941 erhängte sie sich. Ein Opfer der Deportation wurde Hans Herxheimer (18801944). Er war der Neffe des bekannten Frankfurter Dermatologen Karl Herxheimer (1861-1942) und Sohn von Salomon Herxheimer (1841 - 1899). Wie auch Dora Gerson, war er Konvertit, fiel aber genau wie sie durch seine Abstammung unter die im September 1935 erlassenen Nürnberger Rassengesetze und wurde als nicht arisch verfolgt.
Hans Herxheimer war nach seinem Approbationsentzug 1938 bis zu seiner Deportation im Januar 1944 noch als Krankenbehandler tätig. Er verstarb drei Monate nach der Deportierung in Theresienstadt.

Ernst Sklarz, Otto Schlein, Dora Gerson und Hans Herxheimer stehen hier exemplarisch für die unterschiedlichen Lebenswege der jüdischen und jüdischstämmigen Urologen während des Nationalsozialismus. Mit ihnen wird die Geschichte der deutschen Urologie in den 30er- und 40er-Jahren über die bloßen statistischen Zahlen hinweg erlebbar. Die Klärung der Einzelschicksale soll zur Rehabilitierung und Würdigung der betroffenen Urologen beitragen.

Zusammenfassend lässt sich sagen, dass durch den hohen Anteil von $28 \%$ nicht arischen Urologen die deutsche Urologie in ihrer Entwicklung und wissenschaftlichen Arbeit und Lehre starken Veränderungen unterworfen war. Die Ausschaltung und Vertreibung der jüdischen Kollegen hatte weitreichende Konsequenzen, die auch über die Zeit des Nationalsozialismus hinaus zu spüren waren. Der Verlust von fachlich herausragenden Persönlichkeiten wie Leopold Caspar, Hans von Sauer und Alexander von Lichtenberg verzögerte die wissenschaftliche Entwicklung der deutschen Urologie.

\section{Abstract}

\section{The Biographies of Jewish Urologists during National Socialism in Germany}

$\nabla$

During the period of National Socialism (1933-1945) the society of urology, like other medical associations, adjusted quickly to the new political situation. Despite the high proportion of Jewish and Jewish descendants' urologists, this adjustment was nearly without any resistance. With the founding of the Gesellschaft Reichsdeutscher Urologen (GRU), the Gleichschaltung began as early as 1933, consequently ousting Jewish colleagues from practice, research and teaching. Up to now the biographies of the affected urologists are often not well known. Discovering the individual fates of the non-aryan medics is one aspect of researching urology during National Socialism.

\section{Literatur}

1 Krischel M, Moll F, Engels R, Fangerau H. Forschungsperspektiven zur Geschichte der Urologie in Deutschland 1933-1945. Urologe 2010; 49: $1188-1193$

2 Seidler E. Jüdische Kinderärzte 1933 - 1945: entrechtet - geflohen - ermordet. Basel: Karger; 2007: 9-11, 424-425

3 Scholz A, Holubar K, Burg G, Hrsg. Geschichte der deutschsprachigen Dermatologie. Weinheim: WILEY-BLACKWELL; 2009: 131-133

4 Eppinger S. Das Schicksal der jüdischen Dermatologen Deutschlands in der Zeit des Nationalsozialismus. Frankfurt/Main: Mabuse; 2001: $273-287$

5 Schlenzka K, Junge B. Schicksale Magdeburger jüdischer Dermatologen im Nazi-Reich - Carl Lennhoff und Otto Schein. Dermatol Monatsschr 1989; 175: 307-312 